\title{
The Deep Circumflex Iliac Artery Flap for Mandibular Reconstruction and Donor Site Reconstruction with a Patient-Specific Implant: A Case Report
}

\author{
Hyo Joon Kim ${ }^{1}$ and Seong Yong Moon ${ }^{2, *}$ \\ 1 Department of Oral and Maxillofacial Surgery, Chosun University Dental Hospital, Gwangju 61452, Korea; \\ rfa21@naver.com \\ 2 Department of Oral and Maxillofacial Surgery, College of Dentistry, Chosun University, \\ Gwangju 61452, Korea \\ * Correspondence: msygood@chosun.ac.kr
}

Received: 31 January 2020; Accepted: 23 February 2020; Published: 27 February 2020

\begin{abstract}
Jaw defects may occur due to various causes such as tumors, trauma, and diseases caused by infection such as osteomyelitis. Reconstruction of jaw defects has been improved by the free flaps and refined microvascular techniques. The deep circumflex iliac artery (DCIA) flap offers a large amount of bone for complex reconstructions of the mandible. However, several complications and adverse effects can occur, such as abnormal hip contour, hernia, severe bleeding tendency, gait disturbance, and hypoesthesia. To reduce these complications, the monocortical DCIA flap can be used, or iliac crest bone defects can be restored with titanium mesh. However, these methods have limitations when used to reduce hip contour reproduction and gait disturbance. In this study, we report a case of iliac bone reconstruction using a 3D-printed patient-specific implant to reduce donor site morbidity in the reconstruction of the DCIA flap in a patient with oral squamous carcinoma.
\end{abstract}

Keywords: 3D printing; computer-assisted surgery; mandibular reconstruction; DCIA flap; donor site morbidity

\section{Introduction}

Jaw defects may occur due to various causes such as tumors, trauma, and diseases caused by infection such as osteomyelitis. Primary squamous cell carcinoma (SCC) of the mandible is one reason for mandibular resection. Generally, when carcinoma of the mandible invades the skin, the involved soft tissue and mandible are removed and reconstructed using a reconstruction plate and soft tissue flap or complex reconstruction using hard and soft tissue free flaps.

Reconstruction of the jaw requires not only recovery of the contour but also rehabilitation of dentition for chewing and speaking. Therefore, reconstruction of bone and soft tissue through osteocutaneous free flap transfer is regarded as the best treatment to restore function using implants or implant-supported dentures [1].

Hard and soft tissue free flaps used in reconstruction of the jaw include the radial forearm, scapula, fibula, and deep circumflex iliac artery (DCIA) flap [2]. The surgeon chooses the donor site based on various factors such as the size of the hard tissue and soft tissue required for reconstruction and the physical condition of the patient. The DCIA flap has been used with the fibula free flap for the reconstruction of jaw defects because it has the advantages of reproducing the natural shape of the arch, having sufficient width and height, and providing bicortical bone containing good-quality cancellous bone. In particular, it is useful for the restoration of partial defects in the mandible, such as segmental mandibulectomy and hemi-mandibulectomy [3]. Another important factor to consider when choosing donor sites is donor site morbidity. The DCIA is known to have few major complications; however, 
various minor problems have been reported. These complications include gait disturbance, hernia, and fracture. In particular, large bony defects in the ilium area during restoration of a wide bone defect cause weakening of the supporting tissue, which causes complications such as gait disturbance, iliac fracture, and hernia [3]. To reduce this complication, the monocortical DCIA flap can be used, or titanium mesh can be applied to the iliac crest bone defect. However, these methods have limitations when used to reduce hip contour reproduction and gait disturbance [4].

The introduction of Computer-Aided Design (CAD) and Computer-Aided Manufacturing (CAM) systems has led to several advantages for both clinicians and patients, and commercial software can be reliably used to simulate and perform procedures [5].

The purpose of this study was to introduce a case involving reconstruction of the donor site using a patient-specific implant applied after reconstruction of a mandibular defect after segmental mandibulectomy due to primary squamous cell carcinoma in the mandible.

\section{Case Presentation}

We followed the Helsinki Declaration throughout this study. We obtained approval from the Chosun University Institutional Review Board (2-1041055-AB-N-01-2019-08).

A 52-year-old male visited the department of oral and maxillofacial surgery with the complaint that the right lower gingiva was not healed (Figure 1).

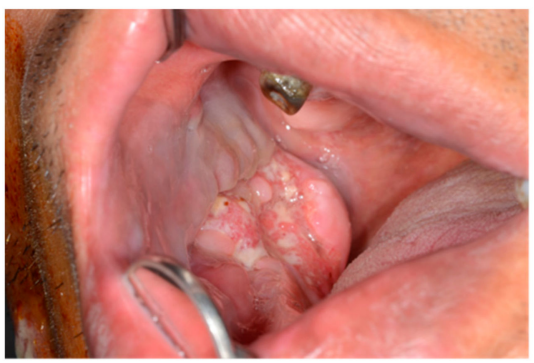

(a)

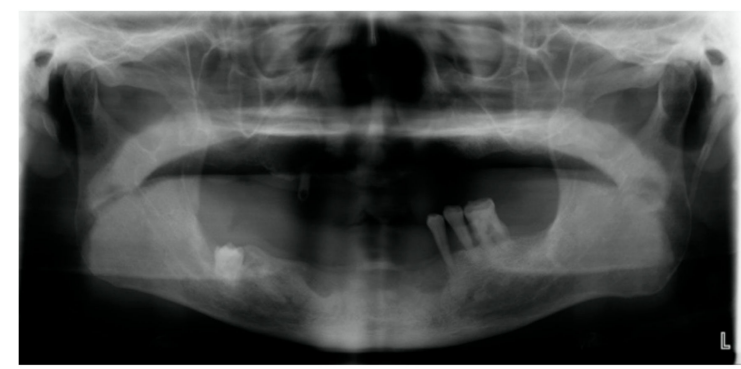

(b)

Figure 1. Initial examinations: (a) Intraoral photograph showing the granulomatous lesion on the right lower gingiva; (b) Panoramic view showing the impacted tooth and osteolytid lesion.

Incisional biopsy results were diagnosed as SCC, and CT and PET-CT images were taken. Osteolytic lesions of the mandible and lymph nodes on the right at level I with intense fluorodeoxyglucose (FDG) uptake were observed on the radiological images. Therefore, segmental mandibulectomy and selective neck dissection (I-V) were planned for the management of the SCC, and use of the DCIA-based iliac crest flap was planned to reconstruct the resected mandibular site.

\subsection{Computer-Assisted Surgical Planning}

For computer-assisted surgery, CT data of the patient's facial bone and ilium were collected. The collected CT data were stored as a dicom file and segmented into mandible and ilium using Mimics software (Materialize, Leuven, Belgium). Segmentation of the lesion site where bone invasion was observed was also performed. The osteotomy line was planned by considering the location of the lesion. The shape of the surgical guide to be used in the actual operation was constructed using Geomagic freeform software (3D Systems, Valencia, USA). The original shape of the mandible and the mirror image of the opposite site were used to shape the bone flap to be reconstructed. The bone flap model was compared with the segmented ilium to select the most suitable shape. The surgical guide for the bone flap was thus prepared (Figure 2). 


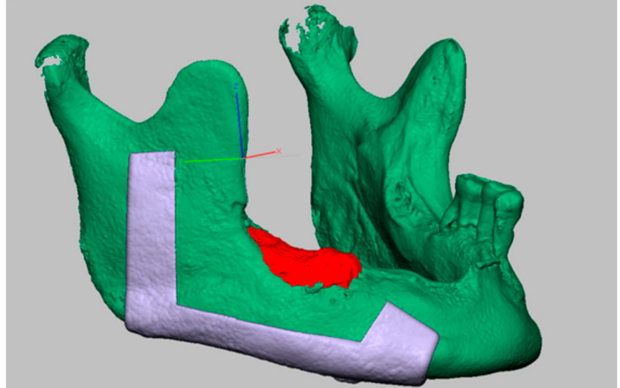

(a)

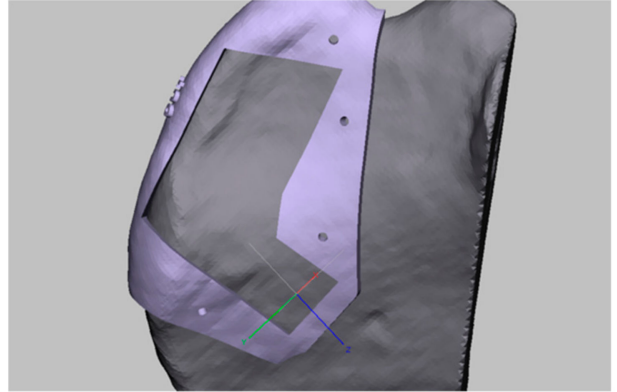

(b)

Figure 2. Computer-based planning and design of surgical guides: (a) Planning and computer-assisted design of the surgical guide for the mandibulectomy; (b) Planning and computer-assisted design of the surgical guide for the iliac crest bone flap.

A customized titanium implant was designed using the shape of the ilium bone flap. The titanium implant reproduces the shape of the ilium while the interior is porous to improve cell proliferation and mesenchymal stem cell differentiation. On the outer surface, a plate shape with a hole for fixation to the ilium was added.

\subsection{Surgical Technique}

The segmental mandibulectomy was performed in the usual way. The osteotomy was performed at the planned location using a prefabricated 3D-printed surgical guide. The DCIA flaps were harvested, including the right iliac crest bone and internal oblique muscle. The iliac crest bone site was osteotomized by applying a 3D-printed surgical guide to the external surface and included an internal oblique muscle of sufficient size (Figure 3).

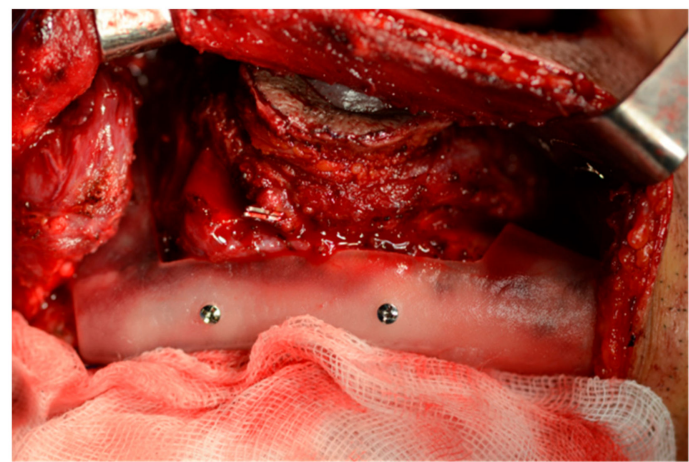

(a)

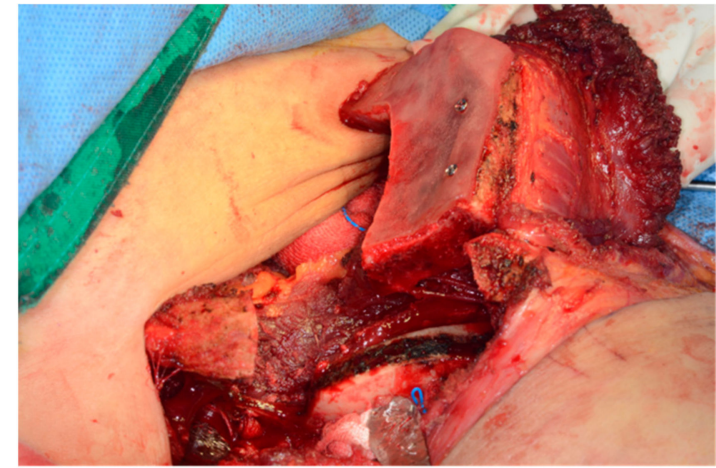

(b)

Figure 3. Intraoperative photograph: (a) The resection guide was applied on the mandible; (b) The iliac crest bone and internal oblique muscle flap was harvested using the resection guide.

Micro-anastomosis with the facial artery and external jugular vein was performed, and fixation was done with a titanium miniplate and screw for reconstruction of the mandible. A preformed 3D-printed porous titanium implant was inserted into the bone defect at the donor site. The titanium implant was fixed to the iliac bone with $2.4 \mathrm{~mm}$ diameter screws through the predesigned hole (Figure 4). 


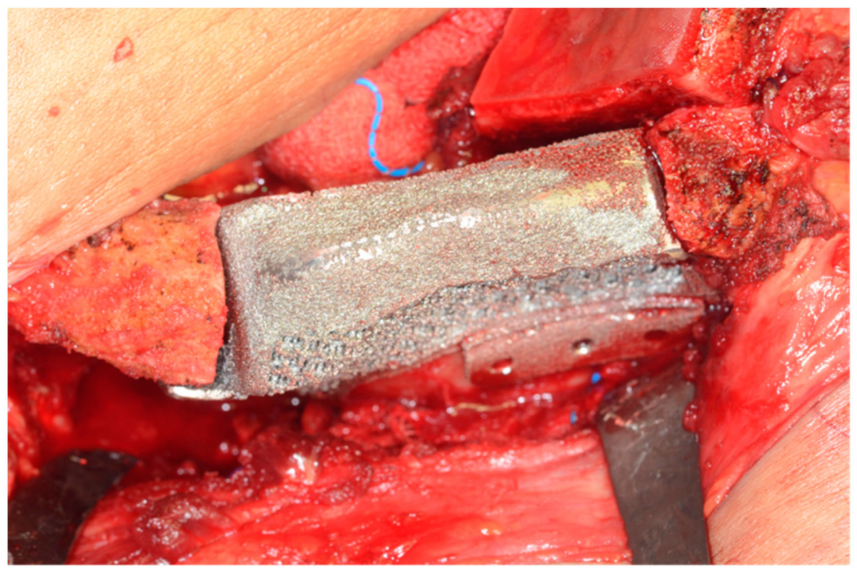

Figure 4. The 3D-printed titanium implant applied for iliac crest reconstruction.

Postoperative CT images were taken, and we fused the 3D model with the preoperative CT. The contours of the mandible and iliac crest were matched between the preoperative and postoperative states (Figure 5).

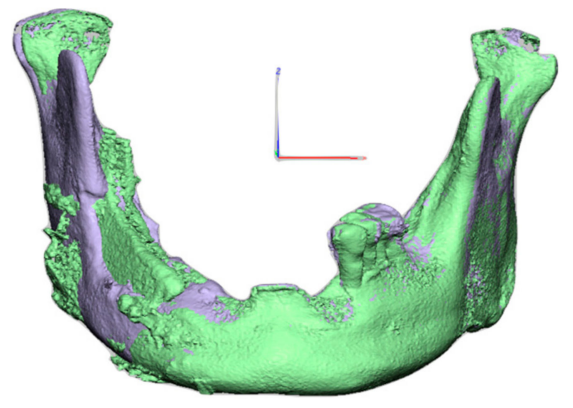

(a)

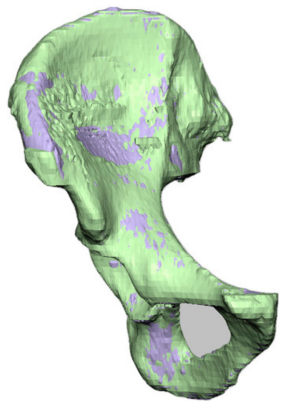

(b)

Figure 5. Fused 3D models for comparison. (a) Purple is the preoperative 3D model, green is the postoperative 3D model of the mandible; (b) Purple is the preoperative 3D model, green is the postoperative 3D model of the iliac bone.

From two weeks post-operation, the patient was able to stand erect and walk, and was able to live without any discomfort up to one year post-operation. No recurrence of the carcinoma or other complication was observed, so implants were placed one year after the operation (Figure 6).

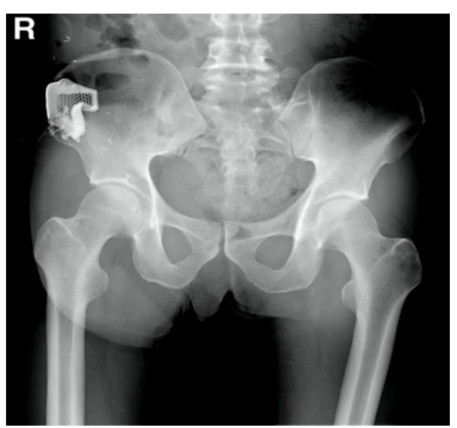

(a)

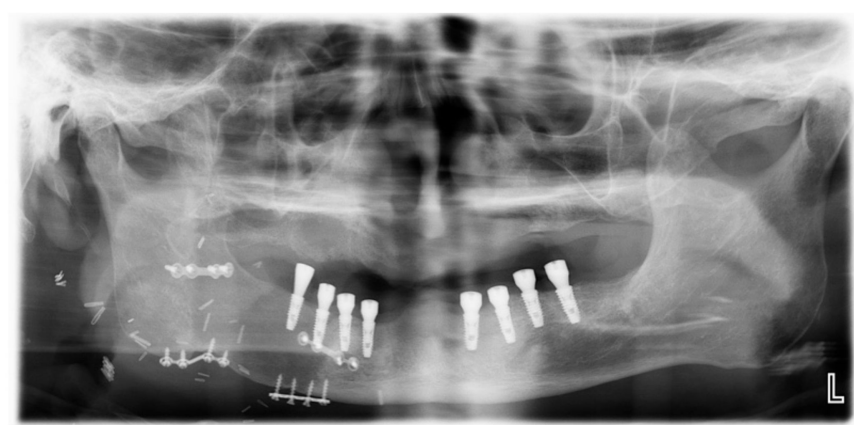

(b)

Figure 6. Postoperative radiographs after one year: (a) Patient-specific implant shown on the right iliac bone after one year; (b) The well-reconstructed mandible; implants were installed one year after the operation. 


\section{Discussion}

With the development of micro-anastomosis techniques and the development of microsurgical instruments [6], reconstruction using free flaps on oral and maxillofacial defects is the best prognostic option. In particular, reconstruction of the mandibular region, in which soft and hard tissues are simultaneously depleted, has been performed using osteocutaneous free flaps or using reconstruction plates and soft-tissue free flaps. Reconstruction using a reconstruction plate and soft-tissue free flap has the disadvantage of causing fracture and exposure of the plate in the long term $[7,8]$. In the case of an osseous free flap, bone union with existing bone tissue can be achieved within 2-3 months and is maintained in the long term through normal bone remodeling [9]. Therefore, a vascularized free flap that comprises hard and soft tissues is mainly used, and reconstruction using a reconstruction plate is becoming a secondary option.

Donor sites containing bone tissue that are mainly used in mandibular reconstruction include the fibula, iliac crest, radial forearm, and scapula. Each donor site differs in the amount of bone available, bone quality and soft tissue, length and thickness of the vascular pedicle, and availability of the two-team approach according to the surgical site. The surgeon has to choose the best flap option for the patient and surgical outcome [10]. The osseous free flap, which has various advantages in the reconstruction of jaw bone defects, has donor site morbidity because it transfers tissues such as hard and soft tissues from the donor site to the defect.

The DCIA-based iliac crest flap was first reported in 1979 by Taylor [11] and Sanders [12]. In 1989, Urken presented a method for reconstructing oral and maxillofacial hard and soft tissue defects using a combination of iliac crest bone and internal oblique muscle. The iliac crest flap has been used for reconstruction of maxillofacial defects because it has good bone quality and sufficient soft tissue [13]. The DCIA-based iliac crest flap has also been reported with various donor site morbidities. Boyd [14] and HS Kim [15] reported herniation through a DCIA donor site. Xiao Feng reported gait disturbance after prolonged sitting and one local contour defect in 1 of 15 DCIA flaps. In this paper, we considered the possibility of such gait disturbance and hip contour defects when reconstructing with the DCIA flap [16].

Research on ways to prevent donor site morbidity that may occur in DCIA flaps has also been conducted in various ways. Minimizing the supportive tissue defect in the iliac area using a monocortical DCIA flap while preserving the ASIS can minimize the complications caused by this bone defect [4]. Iqbal et al. proposed a method to prevent hernia by reconstruction of bone defects using Protack (titanium spiral tacks) and Prolene (polypropylene) mesh [17]. Halsnad reported a method of restoring a donor site using a $0.4 \mathrm{~mm}$ thick titanium sheet [18].

In this report, we proposed reconstruction of the donor site with a 3D-printed titanium implant. This method can alleviate donor complications that may occur in conventional flap reconstruction and can shorten the recovery period. In particular, it is possible to prevent a variety of disorders, such as gait disturbance, loss of hip contour that the patient may feel due to bone defect at the donor site, limitation of mobility, and fracture that may occur at the site weakened by osseous defect. Reconstruction of the iliac bone defect using a 3D-printed patient-specific titanium implant is in accord with the shape of the iliac bone, and we may apply the technique of a surgical guide using computer-assisted surgery. Therefore, the accuracy of the reconstruction is high, which can minimize postoperative complications and shorten the operation time. In addition, the porous structure of titanium implants promotes anchorage with the surrounding bone by improving cell proliferation and mesenchymal stem cell differentiation [19].

Recently, direct reconstruction of maxillofacial defects has been performed using patient-specific 3D-printed implants using medical data and 3D printing techniques [19]. These methods also report satisfactory results and have the advantages of not having to consider additional surgery and donor site morbidities such as the use of free flaps and gait disturbance. This method may be useful for reconstruction of the jaw bone, but the reconstruction of hard and soft tissues requires additional surgery because of the soft tissue coverage. In addition, its use for the restoration of teeth for chewing, 
which is the main function of the jaw, has limitations. Even if the teeth are restored, infection of the titanium implant by the implant teeth may lead to infection of the entire implant. This is a big problem and may require the entire implant to be removed.

On the other hand, reconstruction of jaw defects using hard and soft tissue free flaps and of the donor site using a 3D-printed implant has the following advantages over direct reconstruction.

First, as in this case, it can be used in complex defect cases, including soft tissue defects. Reconstruction of complex defects, such as in this case, requires hard and additional soft tissue at the same time during the operation.

Second, postoperative complications can be prevented. In the case of oral defects, various masticatory muscles and facial expression muscles exist and move for various roles such as emotion expression, mastication, and pronunciation. The oral cavity also contains a variety of microbes due to saliva and external foods, and is more susceptible to infection than other body parts [20]. For this reason, when defects in the oral cavity are directly reconstructed with 3D-printed titanium implants and teeth, various complications may occur, such as exposure of the implant site due to frequent contact, infection, and loosening of the screw fixing the implant to the surrounding bone tissue due to frequent movement. On the other hand, the iliac bone is relatively stable in position and movement due to direct ossification with the remaining bone.

Third, it is easy to recover masticatory function. In the direct reconstruction method, chewing function can be restored through a predesigned titanium implant. However, as discussed above, there is a disadvantage in that it is more vulnerable to infection and it is difficult to change the prosthesis after surgery. This can present a major disadvantage as the oral environment inevitably changes, such as changes in major chewing teeth over time and changes in vertical height. Reconstruction using a free vascularized hard and soft tissue flap allows for prosthetic reconstruction using implant placement like normal oral tissue in a naturally changing environment with the surrounding bone tissue. Removal of the implant, bone graft, and replanting are also possible and safe.

Donor site reconstruction using a 3D-printed patient-specific implant can thus be considered a useful method to reduce donor site morbidity in DICA-based iliac crest flap reconstruction of jaw defects.

Author Contributions: Conceptualization, S.Y.M.; methodology, S.Y.M.; software, S.Y.M., H.J.K.; validation, S.Y.M., H.J.K.; formal analysis, S.Y.M., H.J.K.; investigation S.Y.M., H.J.K.; resources, S.Y.M.; data curation, S.Y.M., H.J.K.; writing—original draft preparation, H.J.K.; writing—review and editing, S.Y.M.; visualization, S.Y.M.; supervision, S.Y.M.; project administration, S.Y.M.; funding acquisition, S.Y.M. All authors have read and agreed to the published version of the manuscript.

Funding: This research was funded by Chosun University Dental Hospital, 2018.

Conflicts of Interest: The authors declare no conflict of interest.

\section{References}

1. Urken, M.L.; Vickery, C.; Weinberg, H.; Buchbinder, D.; Lawson, W.; Biller, H.F. The internal oblique-iliac crest osseomyocutaneous free flap in oromandibular reconstruction. Report of 20 cases. Arch. Otolaryngol. Head Neck Surg. 1989, 115, 339-349. [CrossRef] [PubMed]

2. Schardt, C.; Schmid, A.; Bodem, J.; Krisam, J.; Hoffmann, J.; Mertens, C. Donor site morbidity and quality of life after microvascular head and neck reconstruction with free fibula and deep-circumflex iliac artery flaps. J. Cranio Maxillofac. Surg. 2017, 45, 304-311. [CrossRef] [PubMed]

3. Politi, M.; Toro, C. Iliac Flap Versus Fibula Flap in Mandibular Reconstruction. J. Craniofac. Surg. 2012, 23, 774-779. [CrossRef]

4. Moon, S.-Y. Monocortical Deep Circumflex Iliac Artery Flap in Jaw Reconstruction. J Craniofac. Surg 2015, 26, 1294-1298. [CrossRef]

5. Pagano, S.; Moretti, M.; Marsili, R.; Ricci, A.; Barraco, G.; Cianetti, S. Evaluation of the Accuracy of Four Digital Methods by Linear and Volumetric Analysis of Dental Impressions. Materials 2019, 12, 1958. [CrossRef] [PubMed] 
6. Disa, J.J.; Cordeiro, P.G. Mandible reconstruction with microvascular surgery. Semin. Surg. Oncol. 2000, 19, 226-234. [CrossRef]

7. Schusterman, M.A.; Reece, G.P.; Kroll, S.S.; Weldon, M.E. Use of the AO plate for immediate mandibular reconstruction in cancer patients. Plast. Reconstr. Surg. 1991, 88, 588-593. [CrossRef] [PubMed]

8. Cordeiro, P.G.; Hidalgo, D.A. Soft tissue coverage of mandibular reconstruction plates. Head Neck 1994, 16, 112-115. [CrossRef] [PubMed]

9. Cordeiro, P.G.; Hidalgo, D.A. Conceptual considerations in mandibular reconstruction. Clin. Plast. Surg. 1995, 22, 61-69. [PubMed]

10. Cordeiro, P.G.; Disa, J.J.; Hidalgo, D.A.; Hu, Q.Y. Reconstruction of the mandible with osseous free flaps: A 10-year experience with 150 consecutive patients. Plast. Reconstr. Surg. 1999, 104, 1314-1320. [CrossRef] [PubMed]

11. Taylor, I.G.; Townsend, P.; Corlett, R. Superiority of the deep circumflex iliac vessels as the supply for free groin flaps: Experimental work. Plast. Reconstr. Surg. 1979, 64, 595-604. [CrossRef] [PubMed]

12. Sanders, R.; Mayou, B.J. A new vascularized bone graft transferred by microvascular anastomosis as a free flap. Br. J. Surg. 1979, 66, 787-788. [CrossRef]

13. Urken, M.L.; Vickery, C.; Weinberg, H.; Buchbinder, D.; Biller, H.F. The Internal Oblique-Iliac Crest Osseomyocutaneous Microvascular Free Flap in Head and Neck Reconstruction. J. Reconstr. Microsurg. 1989, 5, 203-214. [CrossRef]

14. Boyd, J.B.; Rosen, I.; Rotstein, L.; Freeman, J.; Gullane, P.; Manktelow, R.; Zuker, R. The iliac crest and the radial forearm flap in vascularized oromandibular reconstruction. Am. J. Surg. 1990, 159, 301-308. [CrossRef]

15. Kim, H.-S.; Kim, J.-Y.; Hur, H.; Nam, W. Herniation after deep circumflex iliac artery flap: Two cases of rare complication. Maxillofac. Plast. Reconstr. Surg. 2016, 38, 10. [CrossRef] [PubMed]

16. Ling, X.F.; Peng, X.; Samman, N. Donor-site morbidity of free fibula and DCIA flaps. J. Oral Maxillofac. Surg. 2013, 71, 1604-1612. [CrossRef] [PubMed]

17. Iqbal, M.; Lloyd, C.J.; Paley, M.D.; Penfold, C.N. Repair of the deep circumflex iliac artery free flap donor site with Protack (titanium spiral tacks) and Prolene (polypropylene) mesh. Br. J. Oral Maxillofac. Surg. 2007, 45, 596-597. [CrossRef] [PubMed]

18. Halsnad, S.M.; Dhariwal, D.K.; Bocca, A.P.; Evans, P.L.; Hodder, S.C. Titanium plate reconstruction of the osseous defect after harvest of a composite free flap using the deep circumflex iliac artery. Br. J. Oral Maxillofac. Surg. 2004, 42, 254-256. [CrossRef] [PubMed]

19. Lee, U.L.; Kwon, J.S.; Woo, S.H.; Choi, Y.J. Simultaneous Bimaxillary Surgery and Mandibular Reconstruction with a 3-Dimensional Printed Titanium Implant Fabricated by Electron Beam Melting: A Preliminary Mechanical Testing of the Printed Mandible. J. Oral Maxillofac. Surg. 2016, 74, 1501.e1-1501.e15. [CrossRef] [PubMed]

20. Keller, W.; Brägger, U.; Mombelli, A. Peri-implant microflora of implants with cemented and screw retained suprastructures. Clin. Oral Implant. Res. 1998, 9, 209-217. [CrossRef] [PubMed]

(C) 2020 by the authors. Licensee MDPI, Basel, Switzerland. This article is an open access article distributed under the terms and conditions of the Creative Commons Attribution (CC BY) license (http://creativecommons.org/licenses/by/4.0/). 